\title{
Cannabis Hyperemesis Syndrome: A Still Under-Recognized Syndrome
}

\author{
Hassene Attout ${ }^{1}$, Sofia Amichi ${ }^{1}$, Françoise Josse ${ }^{2}$, Vincent Appavoupoule ${ }^{2}$, Andry Randriajohany ${ }^{2}$, Yogananda Thirapathi ${ }^{2}$ \\ ${ }^{1}$ Internal Medicine Unit, General Hospital, Ales, France \\ ${ }^{2}$ Internal Medicine Unit, GHER, Saint-Benoît, Reunion
}

Received: $19 / 02 / 2020$

Accepted: 25/02/2020

Published: $27 / 03 / 2020$

How to cite this article: Attout H, Amichi S, Josse F, Appavoupoule V, Randiajohany A, Thirapathi Y. Cannabis hyperemesis syndrome: a still underrecognized syndrome. EJCRIM 2020;7: doi:10.12890/2020_001588.

Conflicts of Interests: The Authors declare that there are no competing interests.

This article is licensed under a Commons Attribution Non-Commercial 4.0 License

\section{ABSTRACT}

Cannabis is one of the most widely used illicit drugs in the world. Its use is associated with several short- and long-term side-effects such as changes in mood, impaired memory, impaired attention, depression and anxiety, and it is correlated with schizophrenia. Cannabinoid hyperemesis syndrome (CHS) is characterized by chronic cannabis use, cyclic intractable nausea and vomiting, and compulsive hot bathing. Patients are typically diagnosed with CHS only after multiple medical evaluations. Recent research has identified type 1 cannabinoid receptors in the intestinal nerve plexus that have an inhibitory effect on gastrointestinal motility. This effect may explain hyperemesis in cannabis users. The thermoregulatory role of endocannabinoids may be responsible for compulsive hot bathing. We describe the cases of two young men seeking repeated emergency room care with recurrent nausea and vomiting. Abstinence from cannabis led to resolution of vomiting symptoms and abdominal pain.

\section{LEARNING POINTS}

- Cannabis is one of the most widely used illicit drugs in world.

- Cannabinoid hyperemesis syndrome is an under-recognized syndrome that can lead to expensive and unrevealing investigations.

- Abstinence from cannabis led to resolution of symptoms.

\section{KEYWORDS}

Cannabis, hyperemesis, hot bathing

\section{CASE DESCRIPTION}

Case 1

A 36-year man presented with recurrent episodes of nausea, refractory vomiting and epigastric pain for 1 week. The previous episode had ended 15 days earlier. These symptoms had begun 3 years previously, occur several times a year and are separated by symptom-free intervals. The patient had presented several times to the emergency department and was usually discharged with symptomatic treatment. His medical history included diabetes mellitus and an episode of acute pancreatitis of alcoholic origin. He had stopped alcohol consumption 5 years earlier. He reported he had smoked five marijuana cigars (each containing $1.0 \mathrm{~g}$ marijuana bud) every day for the previous 10 years. The patient's vital signs were within normal limits and his physical exam was significant only for minor tenderness to palpation in the epigastric region. In particular, no signs of autonomic dysfunction were found. He did not describe a change in bowel habit, antibiotic use, foreign travel or contact with gastroenteritis.

The patient's medical evaluation revealed his complete blood count, basic metabolic profile and hepatic profile were within normal limits with the exception of electrolyte disturbances secondary to protracted emesis. HbA1c was $6 \%$. An abdominal computerized tomography (CT) scan was negative for pathology. Oesophagogastroduodenoscopy showed only mild gastritis. A gastric emptying study revealed mildly delayed gastric emptying. 
The patient also reported having learned to help himself by taking a hot bath each time the symptoms appeared, which dramatically improved his symptoms. Usually, he takes an average of four or five hot baths each day.

The patient was diagnosed with cannabinoid hyperemesis and was advised to avoid cannabis. He decided to undergo withdrawal without the help of psychiatrist.

Currently, there has been no recurrence for 1 year.

Case 2

A 28-year-old man who was a long-term cannabinoid user presented to the emergency department with nausea, vomiting and abdominal pain that had worsened over several days.

His medical history included bipolar disorder. His home medications only included sodium valproate and oxazepam. He had been experiencing similar recurrent episodes for the previous 5 years, frequently associated with life stressors and requiring some previous hospitalizations. In 2017, he had undergone extensive investigation, including laboratory tests, computed tomography of the abdomen, oesophagogastroduodenoscopy and abdominal ultrasound. All tests returned negative for identifiable pathology, with the exception of electrolyte disturbances secondary to protracted emesis. As a result, the patient was diagnosed with gastritis.

On physical examination, the patient's vital signs included a temperature of $37.1^{\circ} \mathrm{C}$, a pulse of 80 beats $/ \mathrm{min}$, a blood pressure of $140 / 80$ $\mathrm{mmHg}$, and a respiratory rate of 18 breaths/min. His abdomen was soft, non-distended and tender.

The patient's medical evaluation revealed that his complete blood count, basic metabolic profile and hepatic profile were within normal limits.

He reported he had smoked three marijuana cigars (each containing $1.0 \mathrm{~g}$ marijuana bud) every day for the last 10 years. Taking hot baths or showers was the most effective means of relieving the symptoms, while antiemetics were ineffective for relieving nausea and vomiting.

The patient was diagnosed with cannabinoid hyperemesis and was advised to avoid cannabis.

The patient was educated on the side-effects of cannabis use and chose to stop cannabis use completely. Currently, there has been no recurrence for 6 months.

\section{DISCUSSION}

Cannabis is one of the most commonly abused drugs worldwide. Its use has dramatically increased over recent years, with production doubling from 1990 to 2006. Additionally, cannabis has become more potent with higher levels of the active ingredient THC being detected. Cannabis also has several medical applications such as use as an antiemetic and an appetite stimulant. More recently, long-term cannabis use has been associated with cyclic episodes of nausea, vomiting and abdominal pain. In 2004, Allen et al. ${ }^{[1]}$ suggested the term 'cannabinoid hyperemesis syndrome' after describing nine patients with a cyclic vomiting illness that began in the setting of long-term cannabis use and resolved after cessation of the drug. Since then several authors have described similar symptoms in their patients ${ }^{[2-4]}$. In 2009 , Sontineni et al. ${ }^{[5]}$ described the important clinical features of CHS, which included long-term cannabis use, cyclic vomiting, colicky abdominal pain, compulsive use of hot showers, and improvement of symptoms with cannabis cessation. Recently, Simonetto et al. ${ }^{[6]}$ proposed major and supportive criteria for the diagnosis of CHS (Table 1). They confirm the essential and major criteria previously proposed by Sontinetti et al. but additionally expand the major criteria and modify the previously proposed 'characteristics suggestive of the diagnosis'.

Cannabinoid hyperemesis syndrome occurs by an unknown mechanism. Despite the well-established anti-emetic properties of marijuana, there is increasing evidence of its paradoxical effects on the gastrointestinal tract and CNS. Tetrahydrocannabinol, cannabidiol and cannabigerol are three cannabinoids found in the cannabis plant with opposing effects on the emesis response. Additionally, it has been proposed that accumulation of the drug leads to delayed gastric emptying ${ }^{[5,6]}$. In order to clarify the causes of cyclic vomiting syndrome which is the main differential diagnosis of CHS, Hejazi et al. ${ }^{[7]}$ performed a retrospective study of 92 adult patients who met Rome diagnostic criteria for cyclic vomiting syndrome. Gastric emptying was measured by gastric scintigraphy. There were 30 patients with a history of cannabis use, 27 patients with a history of migraine, 12 had diabetes mellitus and 10 had irritable bowel syndrome. The subset with delayed emptying was often associated with cannabis use and diabetes mellitus $(p<0.05)$.

The hot-water seeking behaviour of patients is thought to be due to possible deregulation of the thermoregulatory systems of the hypothalamus ${ }^{[6]}$.

It's important to recognize CHS because it may be misdiagnosed as psychogenic vomiting, the cyclic vomiting syndrome, an eating disorder or drug-seeking behaviour, which may lead to extensive and unproductive investigations, psychiatric referrals and ineffective treatments. 


\begin{tabular}{|l|}
\hline Essential for diagnosis \\
\hline Long-term cannabis use \\
\hline Major features \\
\hline Severe cyclic nausea and vomiting \\
\hline Resolution with cannabis cessation \\
\hline Relief of symptoms with hot showers or bath \\
\hline Abdominal pain, epigastric or periumbilical \\
\hline Weekly use of marijuana \\
\hline Supportive features \\
\hline Age less than 50 \\
\hline Weight loss of $>5 \mathrm{~kg}$ \\
\hline Morning predominance of symptoms \\
\hline Normal bowel habits \\
\hline Negative laboratory, radiographic and endoscopic test results \\
\hline
\end{tabular}

\section{REFERENCES}

1. Allen JH, De Moore GM, Heddle R, Twartz JC. Cannabinoid hyperemesis: cyclical hyperemesis in association with chronic cannabis abuse. Gut 2004;11(53):1566-1570.

2. Chang YH, Windish DM. Cannabinoid hyperemesis relieved by compulsive bathing. Mayo Clin Proc 2009;84(1):76-78.

3. Sullivan S. Cannabinoid hyperemesis. Can J Gastroenterol 2010;24(5):284-285.

4. Wallace EA, Andrews SE, Garmany CL, Jelley MJ. Cannabinoid hyperemesis syndrome: literature review and proposed diagnosis and treatment algorithm. South Med J 2011;104(9):659-664.

5. Sontineni SP, Chaudhary S, Sontineni V, Lanspa SJ. Cannabinoid hyperemesis syndrome: clinical diagnosis of an underrecognised manifestation of chronic cannabis abuse. World J Gastroenterol 2009;15(10):1264-1266.

6. Simonetto DA, Oxentenko AS, Herman ML, Szostek JH. Cannabinoid hyperemesis: a case series of 98 patients. Mayo Clinic Proc 2012;87(2):114-119.

7. Hejazi RA, Lavenbarg TH, McCallum RW. Spectrum of gastric emptying patterns in adult patients with cyclic vomiting syndrome. Neurogastroenterol Motil 2010;22(12):12981302 